\title{
Structure-microstructure-property relationships in lead-free BCTZ piezoceramics processed by conventional sintering and spark plasma sintering
}

\author{
Feres Benabdallah ${ }^{1,2,3^{*}}$, Catherine Elissalde ${ }^{2,3}$, U-Chan Chung Seu ${ }^{2,3}$, Dominique Michau ${ }^{2,3}$, Angeline \\ Poulon-Quintin $^{2,3}$, Marion Gayot ${ }^{4}$, Pascale Garreta ${ }^{4}$, Hamadi Khemakhem ${ }^{1}$ and Mario Maglione ${ }^{2,3}$ \\ ${ }^{1}$ Laboratoire des Matériaux Ferroélectriques, Faculté des Sciences de Sfax, Université de Sfax, BP802, \\ 3018 Sfax, Tunisia \\ ${ }^{2}$ CNRS, ICMCB, UPR 9048, F-33600 Pessac, France \\ ${ }^{3}$ Université Bordeaux, ICMCB, UPR 9048, F-33600 Pessac, France \\ ${ }^{4}$ PLACAMAT, UMS 3626 CNRS-Université Bordeaux, F-33600 Pessac, France
}

\begin{abstract}
Lead-free piezoelectric ceramics belonging to the pseudo-binary system (1-x) $\mathrm{BaTi}_{0.8} \mathrm{Zr}_{0.2} \mathrm{O}_{3}-\mathrm{x}$ $\mathrm{Ba}_{0.7} \mathrm{Ca}_{0.3} \mathrm{TiO}_{3}(\mathrm{BCTZ}, \mathrm{x}=0.32$ and 0.5$)$ were prepared using conventional sintering and spark plasma sintering. This comparative study shows how the macroscopic properties of ferroelectric BCTZ perovskites are affected when the grain size is decreased. The crystallographic structure and the microstructural characteristics are thoroughly investigated for ceramic samples obtained by both sintering methods. We clearly demonstrate that the dielectric, piezoelectric, pyroelectric and ferroelectric properties in SPS ceramics are strongly affected by the grain size and the structural defects leading to local strain fields which undermine the polarisation flexibility. This confirms that the critical compositions showing remarkably large piezoelectric coefficients are extremely sensitive to external and internal stresses.
\end{abstract}

Keywords: Lead-free piezoceramics, Spark plasma sintering, ferroelectricity, piezoelectricity, defects

\section{Introduction}

For more than half a century, lead-based piezoelectrics such as $\mathrm{PbZr}_{1-\mathrm{x}} \mathrm{Ti}_{x} \mathrm{O}_{3}(\mathrm{PZT}), \mathrm{Pb}\left(\mathrm{Mg}_{1 / 3} \mathrm{Nb}_{2 / 3}\right)_{1-}$ ${ }_{x} \mathrm{Ti}_{x} \mathrm{O}_{3}(\mathrm{PMN}-\mathrm{PT})$ and $\mathrm{Pb}\left(\mathrm{Zn}_{1 / 3} \mathrm{Nb}_{2 / 3}\right)_{1-\mathrm{x}} \mathrm{Ti}_{x} \mathrm{O}_{3}$ (PZN-PT) have dominated the area of actuator, sensor and transducer technology. These lead-based materials are commonly used with compositions near the morphotropic phase boundary (MPB) where the crystal structure changes abruptly and the electromechanical properties are maximized [1-4].Over the last decade, environmental concerns have been strongly driving the need to replace the lead-based perovskites by equivalent or better performance 
lead-free piezoelectric materials [5-6 ]. Thus, two different approaches have been considered. The first one, the so-called 'compositional engineering approach', focuses on the enhancement of electro-mechanical properties by selecting compositions close to a region with structural instabilities such as polymorphic phase transition (PPT) or MPB [7-10]. The coexistence of different competing phases with equivalent free energies allows a 'softening' of the crystal lattice and an increased alignment of ferroelectric domains during poling (several equivalent polarization orientations at the nanoscale).This gives rise to high piezoelectric effect through polarization rotation or polarization extension mechanisms [11].The second approach is centered on the 'structurally engineered materials' by controlling the micro and/or nanoscale structure. It mainly relies on various structural engineering techniques such as grain size optimization, templated grain growth and domain engineering $[4,12-13]$.

Few years ago, the first approach was successfully used to prepare novel perovskite-structured solid solutions (1-x) $\mathrm{BaTi}_{0.8} \mathrm{Zr}_{0.2} \mathrm{O}_{3}-\mathrm{xBa} 0.7 \mathrm{Ca}_{0.3} \mathrm{TiO}_{3}(\mathrm{BCTZ})$ with a surprisingly large electro-mechanical response surpassing that of the ultrasoft PZT-5H close to room temperature [14]. The unusual properties of the $\mathrm{Pb}$-free pseudo-binary system were attributed to the vanishing polarization anisotropy near a phase convergence region (a nearly spherical degeneration of the free energy surface related to structural instabilities) [15]. Such discovery fostered many researchers to develop even-higher-performance $\mathrm{BaTiO}_{3}$-based materials. Indeed, a modified lead-free piezoelectric $\mathrm{BCTZ}$ with higher Curie temperature $T_{c}$ and colossal piezoelectric activity was designed by increasing the Curie temperature of the two end members in the phase diagram[16]. Moreover, $<001>$ textured BCTZ ceramics ( $B C T Z 50, x=0.5$ ) were synthesized by Ye et al. using templated-grain growth method. The obtained materials were shown to exhibit higher piezoelectric constants and larger electromechanical coupling factors compared to those of randomly oriented BCTZ ceramics whilst $T_{c}$ revealed an increase with the template content [17].

In parallel to the extensive research of new lead free piezoelectrics, the current miniaturization trends in ceramic technology along with the growing consideration for production costs have compelled research efforts to process dense ferroelectric ceramics with both tailored grain sizes and optimal properties using non-conventional methods of synthesis (sol-gel, mechanosynthesis) and sintering such as hot pressing (HP) or spark plasma sintering (SPS). In the latter case, the sintering process allows obtaining high-density ceramics at lower temperatures and shorter processing times (typically a few minutes) compared to conventional sintering. As a result, grain coarsening can be limited. Interestingly, one can mention numerous achievements on the preparation of fine-grained ceramic materials $\left(\mathrm{BaTiO}_{3}, \mathrm{Ba}_{1-\mathrm{x}} \mathrm{Sr}_{x} \mathrm{TiO}_{3}, \mathrm{BaTi}_{1-\mathrm{x}} \mathrm{Zr}_{x} \mathrm{O}_{3}, \mathrm{~K}_{0.5} \mathrm{Na}_{0.5} \mathrm{NbO}_{3}(\mathrm{KNN})\right.$, modified $\mathrm{KNN},(1-\mathrm{x})$ $\mathrm{Bi}_{0.5} \mathrm{Na}_{0.5} \mathrm{TiO}_{3}-\mathrm{x} \mathrm{BaTiO}_{3}$ (BNT-BT),...). Detailed studies have been thus presented elucidating the relationships between the crystallographic structure/grain sizes/domain configurations and their 
ferroelectric /piezoelectric properties [18-24]. However, few available data were reported in the literature emphasizing such correlations in the BCTZ solid solutions, when the grain size is reduced [25-26].

Adjusting the grain size close to $1 \mu \mathrm{m}$ in $\mathrm{BaTiO}_{3}$ ceramics allows to enhance permittivity and piezoelectric coefficient by increasing domain wall density [27]. In addition, a controlled microstructure in terms of defects is also challenging regarding their impact on domain-wall contributions to the dielectric, elastic and piezoelectric properties. Such fine control of the microstructure is hardly achieved using conventionnal sintering as the high sintering temperature required to reach high density promotes grain growth and the long holding time can favor defects. SPS is now well recognized as a very efficient technique to obtain highly densified nanostructured ferroelectric ceramics and this fast consolidation process was largely used to probe the influence of size effects on properties. In this context, in depth investigations of BCTZ piezoceramics with selected compositions and controlled grain size using advanced sintering technique are still required for a better understanding of the relationships between structure, microstructure and functional properties. We propose a comparative study of BCTZ ceramics (compositions $x=0.32$ and 0.5 ) processed by conventional sintering and SPS. The aim is to probe the impact of the microstructure on the dielectric, pyroelectric, ferroelectric and piezoelectric responses of BCTZ ceramics.

Structural defects induced by the SPS process were linked to a lower domain wall mobility which could explain the altered piezoelectric and ferroelectric performances of the BCTZ compositions sintered by SPS.

\section{Experimental}

The perovskite-structured BCTZ powders with a generic formula (1-x) $\mathrm{BaTi}_{0.8} \mathrm{Zr}_{0.2} \mathrm{O}_{3}-\mathrm{xBa}_{0.7} \mathrm{Ca}_{0.3} \mathrm{TiO}_{3}$ $(x=0.32$ and 0.5$)$ were synthesized by conventional solid state reaction method using as starting chemicals, $\mathrm{BaCO}_{3}$ (99.95\%), $\mathrm{CaCO}_{3}$ (99.5\%), $\mathrm{ZrO}_{2}$ (99.99\%) and $\mathrm{TiO}_{2}$ (99.9\%). For each composition, the raw materials were weighed according to the stoichiometric formula and mixed by ball milling in alcohol using zirconia balls for $4 \mathrm{~h}$. After being dried, the mixtures were calcinated in oxygen atmosphere at $1350^{\circ} \mathrm{C}$ during $15 \mathrm{~h}$, then ball milled again to break the agglomerates. Two sintering methods were used to obtain dense BCTZ ceramics with different grain sizes and morphologies. For spark plasma sintering, the powders were loaded in a cylindrical graphite die with an inner diameter of $10 \mathrm{~mm}$ and heated under vacuum using an SPS apparatus (SYNTEX. INC, SPS-515S). The rapid heating was achieved owing to a pulsed direct electric current traversing the die. The temperature was raised at $50-100^{\circ} \mathrm{C} / \mathrm{min}$ and kept at a constant value of $1300^{\circ} \mathrm{C}$ for $10 \mathrm{~min}$. Afterwards, the sample was cooled to $600^{\circ} \mathrm{C}$ with a relatively low cooling rate of $35^{\circ} \mathrm{C} / \mathrm{min}$ and then, to room temperature by shutting down the power supply. A constant pressure of $90 \mathrm{MPa}$ was applied along 
the Z-axis of the graphite die during the whole sintering process. Finally, the SPS-processed samples were annealed in oxygen at $900-1000^{\circ} \mathrm{C}$ for $12 \mathrm{~h}$ in order to remove surface carbon contamination and to limit oxygen vacancies caused by the reducing conditions. For the conventional sintering, the powders were pressed into disks $(\Phi=8 \mathrm{~mm}, \mathrm{e}=1 \mathrm{~mm})$ under a uniaxial pressure of $120 \mathrm{MPa}$ and sintered at $1450^{\circ} \mathrm{C}$ for $4 \mathrm{~h}$ under oxygen atmosphere.

Phase structure of the BCTZ powders and ceramics was identified by X-ray powder diffraction (XRD) using a PANalytical X'Pert MPD diffractometer with Cu Ka radiation of wavelength $1.54056 \AA$. The XRD patterns were recorded at room temperature with scan rates ranging from 0.02 to $0.008^{\circ}$ and a counting time of $70 \mathrm{~s} / \mathrm{step}$. The microstructures of the ceramic samples were observed using a scanning electron microscope (SEM, JEOL $6360 \mathrm{~A}$ ) and a transmission electron microscope (TEM-FEG, JEOL-JEM-2200FS with an acceleration voltage of $200 \mathrm{kV}$ ). Fractures of SPS ceramics (BCTZ32) were thermally etched at $1200^{\circ} \mathrm{C}$. The samples were introduced in the hot region of the tube furnace, kept at $1200^{\circ} \mathrm{C}$ during $1 \mathrm{~h}$ and then quenched. BCTZ50 ceramics were chemically etched in HCL for $4 \mathrm{~h}$. The grain size of the sintered ceramics was evaluated by linear intercept method on scanning electron microscopy micrographs. The average grain size was evaluated for each sample based on at least 100 grains. Thin foils for TEM were prepared by mechanical polishing to a thickness of $80 \mu \mathrm{m}$ and argon ion-beam polishing using a 691 Gatan Precision argon Ion Polishing System (PIPS, 5kV).

For electrical measurements, both sides of polished ceramic disks were sputter coated with gold electrodes. The temperature dependence of the dielectric constant and losses were measured at different frequencies $\left(10^{2}-10^{5} \mathrm{~Hz}\right)$ using an LCR meter (Wayne Kerr 4300) connected to a hightemperature tube furnace. The polarization-electric field $(\mathrm{P}-\mathrm{E})$ hysteresis loops were recorded at room temperature based on a standard Sawyer-Tower circuit. Samples for pyroelectric and piezoelectric measurements were poled under various DC electric fields $(\approx 10-15 \mathrm{kV} / \mathrm{cm})$ during cooling from the ferroelectric-non ferroelectric phase transition temperature down to the lowtemperature region where a stable ferroelectric phase with rhombohedral symmetry was evidenced for both BCTZ compositions. The piezoelectric responses for the radial mode were determined at room temperature by the resonance-antiresonance method on the basis of IEEE standards [28]. The thermal variation of the pyroelectric current and the spontaneous polarization $\mathrm{P}_{\mathrm{s}}$ was investigated for all samples by using a digital multimeter Keithley 2100 .

\section{Results and discussion}

\subsection{Microstructural characterizations}


Scanning electron microscope (SEM) images of fracture surfaces illustrate the microstructural features of the BCTZ ceramics prepared by conventional and spark plasma sintering methods (Figs. $1 a-f)$. All the samples are well densified with almost no noticeable large porosity. The bulk relative densities of BCTZ32 and BCTZ50 obtained by conventional sintering were found to be respectively 93\% and $90 \%$. Whatever the composition, both intergranular and intragranular fracture modes are observed. The inhomogeneity of the microstructures arises from exaggerated grain growth occurring at the high sintering temperature $\left(1450^{\circ} \mathrm{C}\right)(\mathrm{Fig} .1 \mathrm{a}, 1 \mathrm{~b})$. The average grain size and its standard deviation depend on the chemical composition of the ceramics, ranging between $10 \pm 4.0 \mu \mathrm{m}$ for BCTZ32 and $15 \pm 6 \mu \mathrm{m}$ for BCTZ50. In both compositions, few grains with a size 25-30 $\mu \mathrm{m}$ are observed.

When the sintering temperature was decreased using SPS $\left(150^{\circ} \mathrm{C}\right.$ lower than for conventional sintering), the relative densities significantly increased and reached values close to $97 \%$ and $98 \%$ for $\mathrm{BCTZ32}$ and BCTZ50 respectively. No further increase of the density was noticed when the pressure during SPS sintering is raised from $90 \mathrm{MPa}$ to $110 \mathrm{MPa}$. SPS ceramics exhibit complex fractured surface with predominantly intragranular fracture mode. The grain size determination was possible only after revealing grain boundaries by thermal and chemical etching according to the samples. Whatever the composition, finer microstructures are observed compared to conventional sintering (Figs. 1c-f). The average grain size is $2 \pm 1 \mu \mathrm{m}$ for BCTZ32 and decreased in the sub-micrometer range $0.8 \pm 0.4 \mu \mathrm{m}$ for BCTZ50. The grains are mainly equiaxed and few spherical shaped grains can be distinguished among the smallest ones. As expected, the microstructural investigations confirm that enhanced kinetics during SPS allow to retain fine grain microstructure in BCTZ ceramics.

TEM micrographs of the BCTZ32 pellet sintered by SPS are shown in fig 2 . The images reveal the existence of numerous isolated dislocations within some individual grains (located in a region ranging from 300 to $400 \mathrm{~nm}$ close to the grain boundaries) witnessing of a plastic activity inside the grains. Evidence of such defects clearly shows that SPS process performed under selected parameters of temperature and pressure, can induce thermo-mechanical solicitations at the top surface of grains during sintering leading to their plastic deformations.

This unusual activity at the nanoscale has not been detected for all grains of the ceramic probably due to the random orientation of the crystallites with respect to the SPS pressure axis. A more detailed study would be required to examine how the sizes and/or densities of dislocations change as a function of the operating parameters during the sintering process. 


\subsection{Crystallographic analysis}

Room temperature XRD patterns of polycrystalline $B C T Z(x=0.32$ and 0.5$)$ prepared using both sintering methods are shown in fig.(3-a,3-b). All samples have single-phase perovskite structure within the sensitivity of the XRD technique. However, one should emphasize the distinct structural trends for different processing conditions.

For $\mathrm{BCTZ50}$ sintered at $1450^{\circ} \mathrm{C} / 4 \mathrm{~h}$, the powder pattern was indexed in the tetragonal symmetry with space group $P 4 \mathrm{~mm}$. Nevertheless, recent XRD investigations with synchrotron radiation have allowed to identify an intermediate ferroelectric phase (orthorhombic, Amm2 space group) extending over a relatively broad temperature range (260-300K) between two other ferroelectric phases of rhombohedral $(R 3 m)$ and tetragonal $(P 4 m m)$ symmetry [15]. This result is consistent with the recent work of Zhang et al. highlighting the presence of a line of high-performance compositions in the BCTZ system undergoing a tetragonal - orthorhombic phase transition at room temperature[29]. The volume fractions of the coexisting phases and their lattice parameters in the case of BCTZ50 determined from Rietveld refined fits to the data at $300 \mathrm{~K}$ are listed in table $\mathrm{I}$.

\begin{tabular}{|c|c|}
\hline $\mathrm{BCTZ50}(x=0.5)$ & $\begin{array}{l}\text { Rietveld refined structure obtained } \\
\text { at } 300 \mathrm{~K}\end{array}$ \\
\hline Standerd XRD $(\lambda=1.54056 \AA)$ & $\begin{array}{l}{ }^{*} P 4 m m, a=b=4.000(5) \AA \\
C=4.0173(3) \hat{A} \\
\alpha=\beta=\gamma=90^{\circ} / / \text { volume } \\
\text { fraction }=100 \%\end{array}$ \\
\hline High-resolution synchrotron XRD [15] & $\begin{array}{l}{ }^{*} \text { Amm } 2, a=3.9992(6) \hat{A}, \\
b=5.6683(0) \AA \quad A=5.6774(4) \AA \\
\alpha=\beta=\gamma=90^{\circ} / / \text { volume } \\
\text { fraction }=66.496 \%\end{array}$ \\
\hline$(\lambda=0.35419 \AA)$ & $\begin{array}{l}{ }^{*} P 4 m m, a=b=3.9995(1) \AA \\
c=4.0191(6) \hat{A} \\
\alpha=\beta=\gamma=90^{\circ} / / \text { volume } \\
\text { fraction }=33.504 \%\end{array}$ \\
\hline
\end{tabular}

As for BCTZ32 prepared by conventional sintering, all the diffraction peaks of the powder pattern (using the standard XRD technique) were indexed in the cubic symmetry at $300 \mathrm{~K}$ ( $\mathrm{Pm} \overline{3} \mathrm{~m}$ space group, $a=4.0206(2) \AA, \alpha=\beta=\gamma=90^{\circ}$ ). Full Rietveld refinements were performed as well using synchrotron radiation and the crystal structure was found to be cubic ( $a=4.0216(9) \AA$, $\left.\alpha=\beta=\gamma=90^{\circ}\right)$. These results are not compatible with the piezoelectricity of the material at room temperature. However, it is worth noting that BCTZ32 is located in the vicinity of a phase convergence region' where a cubic non-ferroelectric phase merges with three ferroelectric phases of rhombohedral, orthorhombic and tetragonal symmetry without breaking the Gibbs phase rule in the 
$\mathrm{BaTi}_{0.8} \mathrm{Zr}_{0.2} \mathrm{O} 3-\mathrm{Ba}_{0.7} \mathrm{Ca}_{0.3} \mathrm{TiO}_{3}$ pseudo-binary phase diagram [15]. Thus, it becomes difficult to provide evidence of such structural distortions away from the parent cubic structure.

Several structural evolutions have been observed for the samples sintered by SPS at $1300^{\circ} \mathrm{C} / 90 \mathrm{MPa}$ /10 min. First, the diffraction peaks for BCTZ50 ceramic are shifted to the higher diffraction angles revealing the shrinkage of the unit cell volume. Such behaviour attributed to an increase in the internal stress in the ceramic is less pronounced for BCTZ32. Furthermore, the splitting of $(200)_{p c}$ Bragg reflection characteristic of a tetragonal lattice distortion (P4mm space group) in coarse-grained BCTZ50 sample is transformed into a broad single peak typical of a pseudo-cubic symmetry in finegrained one. This suggests a progressive reduction of the long range ferroelastic strain and the intrinsic unit cell polarization with decreasing grain size. Similar trends have been reported for the niobium-doped PZT ceramics by C. A. Randall et al.[30].

The broadening of all diffraction profiles detected for the SPS processed samples can be correlated with the existence of microstrains which may originate from a spreading of dislocations within the individual grains as observed in TEM. In general, elastic fields resulting from structural defects could introduce a contribution to the width of each reflexion [31].

\subsection{Low-frequency impedance spectroscopy measurements}

Figure 4 shows the temperature dependence of the dielectric constant $\varepsilon_{r}$ and loss tangent $(\tan \delta)$ of the BCTZ ceramics under investigation recorded at $10 \mathrm{kHz}$. For the SPS sintered samples, the relative dielectric permittivity drastically decreases at the phase transition temperature $\left(T_{m}\right)$ which shifts to higher values for both compositions. This significant permittivity drop may be attributed to the increasing contribution of the non-ferroelectric low-permittivity grain boundaries as a consequence of the dielectric mixing(brick-wall model) [32-33].The dielectric constant peaks become much broader leading to a more pronounced diffuse phase transition (DPT) behaviour, already present in coarse grained ceramics. The dielectric permittivity is moderately rising at the low temperature regime while the loss tangent increases over the whole temperature range with decreasing grain sizes. This temperature dependence of dielectric properties is attributed to the contribution of extrinsic effects. The abnormal broadening of the dielectric peaks can be explained by an increase of both compositional fluctuations and strain resulting from the change in microstructure of the SPS ceramics compared to the conventional ones. Such interpretation is well supported by the work of Wu et al. reporting the peak width at two-third maximum dielectric constant as a linear function of the compositional fluctuations [34]. Besides, it is important to underline that the sequence corresponding to rhombohedral-orthorhombic (R-O) and orthorhombic-tetragonal (O-T) phase 
transitions is no longer obvious in SPS processed BCTZ50 ceramics due to the increase of the diffuse character of the permittivity maximum (Fig $4 a$ and inset of Fig $4 b$ ).

To provide a clear description of the phase transition behaviour for SPS BCTZ samples, the temperature dependences of the dielectric constant and losses were measured at different frequencies ranging between $100 \mathrm{~Hz}-100 \mathrm{kHz}$. As shown in figure 5c, BCTZ50 exhibits a diffuse phase transition with a broad peak at the corresponding dielectric temperature $\left(T_{m}\right)$, with no apparent shift of this maximum over the investigated range of frequency. On the other hand, BCTZ32 displays a strongly broadened dielectric anomaly at $T_{m}$ which shifts to higher values with increasing frequency, thus suggesting the occurrence of a relaxor state (Fig.5a).

The dielectric losses notably increase with increasing frequency at low temperature regime for both compositions (Figs. 5a-c).We ascribe the observed frequency dispersion of the dielectric response to the presence of additional extrinsic contributions to polarization processes associated with the dynamics of polar domains and randomly distributed defects. At high temperatures, the increase of $\tan \delta$ at low frequency values is attributed to the thermal activated conductivity due to the motion of oxygen vacancies.

To further confirm the peculiar dielectric trends of BCTZ samples obtained by SPS process, we use the modified Curie-Weiss law proposed by K. Uchino and Nomura [35]:

$$
\frac{1}{\varepsilon_{r}}-\frac{1}{\varepsilon_{r m}}=\frac{\left(T-T_{m}\right)^{\gamma}}{C} \quad \text { Eq.1 }
$$

where $\varepsilon_{r m}$ is the maximum value of dielectric constant at the phase transition temperature $T_{m}, Y$ is the degree of diffuseness, and $C$ is the Curie-like constant. The limiting values $\gamma=1$ and $\gamma=2$ correspond to the case of normal ferroelectric and ideal relaxor ferroelectric respectively. Figures $5 \mathrm{~b}$ and $5 \mathrm{~d}$ show the plots of $\ln \left(\frac{1}{s_{r}}-\frac{1}{s_{r m}}\right)$ as a function of $\ln \left(T-T_{m}\right)$ at $100 \mathrm{kHz}$ in the temperature range $T>T_{m}$. A linear (power-law) relationship is obvious for all compositions. The fitted $\gamma$ values are 1.98 and 1.64 for BCTZ32 and BCTZ50 respectively (while it is found to be 1.73 and 1.53 for ceramics with the same compositions obtained by conventional sintering). Such increased values reflect a strengthening of the relaxor character from standard to SPS sintering and from BCTZ50 to BCTZ32.

At this stage, one can gives emphasis to the close relationship between the structural/ microstructural features of the BCTZ ceramics and their dielectric responses. From Transmission electron microscope (TEM) and X-ray powder diffraction (XRD) analysis, it is clear that the spark plasma sintering process can induce structural disorder due to both grain size decrease and local 
strain field increase. As a result a gradual transformation is observed from ferroelectric DPT behaviour (conventional sintered BCTZ32) towards a proper relaxor state (SPS sintered BCTZ32).

However, we should underline that SPS may intensify the chemical disorder (compositional inhomogeneity) as well, for example through an increased density of Zr-rich clusters because of the fast sintering kinetics. These large compositional fluctuations are correlated to the fluctuation of the cell parameters and as a result, have pronounced effects on the dielectric properties of the perovskite system.

\subsection{Ferroelectric and piezoelectric properties}

The temperature dependence of the spontaneous polarization $\mathrm{P}_{\mathrm{s}}$ is given for each SPS processed $\mathrm{BCTZ}$ ceramic in figure 6. It is clear that $\mathrm{P}_{\mathrm{s}}$ decreases smoothly through the dynamic transition temperature $T_{m}$ and retains finite values up to rather high temperatures because polar domains persist well above $T_{m}$. The latter physical picture is typical of a diffuse phase transition (BCTZ50) which may progressively be forced to merge into a relaxor state (BCTZ32) with a higher degree of chemical /structural disorder. These findings confirm that the already smooth temperature variation of the polarization reported in standard ceramics is even more softened in SPS ceramics [36].

The ferroelectric switching behaviour was investigated in terms of the polarization-versus electricfield hysteresis loops measured at $50 \mathrm{~Hz}$ and at room temperature. As shown in figure 7; the P-E loops recorded for the conventionally sintered ceramics are slim giving a small remanent polarization and a relatively low coercive field. The $P_{r}$ and $E_{c}$ values were found to be $3 \mu \mathrm{C} . \mathrm{cm}^{-2}, 0.975 \mathrm{kV} . \mathrm{cm}^{-1}$ for BCTZ32 and $5.6 \mu \mathrm{C} . \mathrm{cm}^{-2}, 2.75 \mathrm{kV} \mathrm{cm}^{-1}$ for BCTZ50. However, the SPS processed ceramics exhibit well saturated hysteresis loops and the measured ferroelectric responses are strongly weakened giving smaller $\mathrm{P}_{\mathrm{r}}$ and higher $\mathrm{E}_{\mathrm{c}}\left(2.8 \mu \mathrm{C} . \mathrm{cm}^{-2}\right.$ and $4 \mathrm{kV} . \mathrm{cm}^{-1}$ respectively for BCTZ50). Such polarization-field dependences with decreasing grain size appear to be a general trend for both $\mathrm{Pb}$-based and $\mathrm{Pb}$-free perovskite-structured solid solutions [30, 37-39].

The critical change in the ferroelectric switching characteristics can be interestingly explained using two different and complementary approaches. The first one involves the theory of D-E hysteresis loops based on the Avrami model proposed by Orihara [40]: the proportion of grains contributing to polarization reversal mechanism is given by;

$$
f=f_{0}\left[1-\exp \left(-\frac{G_{a} d^{\mathrm{s}}}{k T}\right)\right]
$$


where $G_{a}$ represents the grain anisotropy energy density and $d$ is the grain size. Thus, it is well understood that this proportion decreases in fine grained ceramics leading to the drop of the ferroelectric properties.

The second approach, of great relevance for the ferroelectric materials, is related to the interaction of defects with domain walls. These defects are, generally, either intrinsic or induced by the addition of dopants. In this study, it is concluded that the existence of dislocations with a significant spatial extent and isolated charged defects (oxygen vacancies) in SPS ceramics acts as strong pinning centers to inhibit domain-wall mobility [41]. Moreover, when the grain size decreases, increasing the number of grain boundaries will systematically increase the pinning points for the moving walls. As a result, the macroscopic ferroelectric properties are affected as follow:

(1) The values of remanent and saturated polarization decline because of the reduction of the achievable domain alignment with the applied electric field.

(2) A drastic drop in the domain wall motion meaning that higher switching fields are required during the polarization reversal process. From the thermodynamic point of view, the structural defects locally modify the ferroelectric double-well potential depth resulting in higher barrier energy for the motion of the domain wall [41, 42].

The piezoelectric resonance measurements were performed at room temperature using an HP4194 A impedance analyzer on ceramic samples prepared in disc shapes. The complex admittance (inverse of the impedance $Z$ ) of the resonator was recorded as a function of the frequency across the first resonance (the fundamental resonance) corresponding to the radial vibration mode. The experimentally obtained data of the real (conductance $\mathrm{G}$ ) and imaginary (susceptance B) components of the admittance were then fitted to the theoretical spectra generated from the admittance equation of an equivalent circuit model [a Van Dyke model with real circuit parameters: $C_{0}, R_{0}$ (for the static branch) and C, L, R (for the motional branch)]. This model is recommended by the IEEE standard on piezoelectricity $[28,43-46]$. The use of a nonlinear least-squares fit to the equivalent circuit model allows us to find the values of the circuit constants $C_{0}, R_{0}, R, L$ and $C$. The material constants such as the transverse piezoelectric coefficient $d_{31}$, the electro-mechanical coupling factor $K_{p}$, the mechanical quality factor $Q_{m}$ can then be directly determined from the circuit parameters and the values of the parallel and series resonance frequencies $\left(f_{p}\right.$ and $\left.f_{s}\right)$ given by:

$$
\begin{array}{r}
f_{s}=\frac{1}{2 \pi \sqrt{L C}} \quad \text { Eq.3 } \\
f_{p}=f_{s} \sqrt{1+\left(\frac{C}{C_{0}}\right)} \quad \text { Eq.4 }
\end{array}
$$


Figure 8 shows the recorded spectra of the conductance $G$ and the susceptance $B$ around the radial vibration mode for poled BCTZ50 ceramic discs (both normal and SPS sintered samples) along with their corresponding admittance locus (G-B curves). The electrical behaviour of the free piezoelectric resonator was fully determined in accordance with the previously mentioned protocol. The values of $d_{31}, K_{p}$ and $Q_{m}$ were found to be $197 \mathrm{pC} / \mathrm{N}, 0.45$ and 77 respectively for normal sintered sample, whereas, the SPS processed one revealed inferior piezoelectric coefficients with values as low as $\mathrm{d}_{31}=43 \mathrm{pC} / \mathrm{N}, \mathrm{K}_{\mathrm{p}}=0.12\left(\mathrm{Q}_{\mathrm{m}}\right.$ is raised to a value as high as 334$)$. Similar trends were unambiguously disclosed for BCTZ32 composition, showing decreasing values of the electromechanical coefficients from about $d_{31}=120 \mathrm{pC} / \mathrm{N}, \mathrm{K}_{\mathrm{p}}=0.33, \mathrm{Q}_{\mathrm{m}}=106$ using the conventional sintering technique to $30 \mathrm{pC} / \mathrm{N}, 0.1$ and 200 respectively using SPS.

Similarly to the dielectric and ferroelectric properties, it is understandable that the electromechanical responses should be correlated to the structural /microstructural features in BCTZ ceramics: the excellent piezoelectric properties obtained for conventionally sintered BCTZ50 ceramic originate from the different coexisting/competing phases $(\mathrm{Amm} 2+\mathrm{P} 4 \mathrm{~mm})$ close to room temperature. The material was shown to be quite 'soft' with respect to the electric field (easy paths for polarization rotation in anisotropically flattened free energy profile) and, even more importantly, with respect to the crystal lattice (large electrostrain obtained under small electric field)[47-48]. These two effects go hand in hand leading to high piezoactivity due to faster domain wall motion under external stresses. Here, it is important to keep in mind the underlying structural mechanism stressing the key role of domain structures for the $\mathrm{Pb}$-free $\mathrm{Ba}\left(\mathrm{Zr}_{0.2} \mathrm{Ti}_{0.8}\right) \mathrm{O}_{3}-\mathrm{x}\left(\mathrm{Ba}_{0.7} \mathrm{Ca}_{0.3}\right) \mathrm{TiO}_{3}$ system: experimental evidences are presented showing that $d_{33}$-maximum region is tied strictly to a nanometer-sized ferroelectric domains with a domain hierarchy in the 'morphotropic phase boundary, MPB' regime [49]. In SPS ceramics, additional extrinsic effects arising from the existence of structural defects (dislocations) and point defects (oxygen vacancies) leading to local strain fields hinder the domain wall mobility and thus alter electromechanical efficiency.

\section{Conclusions}

Dense BCTZ ferroelectric ceramics of the pseudo-binary system (1-x)BaTi $\mathrm{Ba}_{0.8} \mathrm{Zr}_{0.2} \mathrm{O}_{3^{-}} \quad \mathrm{x}$ $\mathrm{Ba}_{0.7} \mathrm{Ca}_{0.3} \mathrm{TiO}_{3}(\mathrm{x}=0.32,0.5)$ have been successfully prepared using both conventional and spark plasma sintering techniques. The effects of their crystallographic structures along with microstructural characteristics on the corresponding dielectric, ferroelectric and piezoelectric properties have been studied in detail. Drastic changes have been evidenced when decreasing the grain size for the selected BCTZ compositions using SPS. This behaviour was in part explained by the existence of microstrains or more generally by a random distribution of defects generating random elastic fields. 
In comparison with coarse BCTZ ceramics, the low-frequency dielectric measurements reveal a significant drop of the dielectric constant at the phase transition temperature in fine-grained samples. It was also shown that the SPS process leads to enhanced DPT behaviour which can gradually be transformed into a relaxor state in the case of BCTZ32. Such property tuning is systematically driven by structural disorder and composition fluctuations.

The improved ferroelectric and piezoelectric properties observed in conventional sintered BCTZ ceramics are linked to the different coexisting phases at the nanoscale near the room temperature. The vanishing polarization anisotropy near a so-called 'phase convergence region', the lattice softening and the consequent high domain sensitivity to external stresses are considered as the main features of the (1-x) $\mathrm{BaTi}_{0.8} \mathrm{Zr}_{0.2} \mathrm{O}_{3}-\mathrm{x} \quad \mathrm{Ba}_{0.7} \mathrm{Ca}_{0.3} \mathrm{TiO}_{3}$ solid solutions processed using conventional sintering method. These colossal responses go down in SPS ceramics due to the lower domain mobility resulting from the existence of microstructural defects. Our main conclusion is that the BCTZ compositions under investigation display large piezoelectric coefficients because their polarization is highly sensitive to moderate external stress. This is why their ferroelectric properties are affected by the microstructural distortions resulting from the SPS sintering while other ferroelectric materials like pure $\mathrm{BaTiO}_{3}$ require much stronger disturbances. In terms of apparent size effects, we have evidenced large changes in the ferroelectric properties for average grain sizes ranging between $\approx 0.8$ $2 \mu \mathrm{m}$, while shorter length scale $(\approx 100 \mathrm{~nm})$ should be reached in the case of pure $\mathrm{BaTiO}_{3}[50]$.

\section{Acknowledgment}

F. Benabdallah and co-authors are all grateful for financial support by the French National Center for Scientific Research (CNRS) and the ministry of higher education and scientific research of Tunisia. We thank also Annie Simon for fruitful discussions, Sonia Gomez for SEM observations and Eric Lebraud for XRD measurements. The authors acknowledge PLACAMAT (UMS 3626 CNRS-University Bordeaux) for technical support.

\section{References}

[1] Setter N. Piezoelectric Materials and Devices. Ceramics Laboratory, EPFL Swiss Federal Institute of Technology 2005; Lausanne.

[2] Moulson A J, Herbert J M. Electroceramics: Materials, Properties and Applications. John Wiley \& Sons Ltd. 2003; New York.

[3] Sebald G, Lebrun L, Guiffard B, Guyomar D. Morphotropic PMN-PT system investigated through comparison between ceramic and crystal. J Eur Ceram Soc 2005; 25: 2509-2513 
[4] Park S-E, Shrout T R. Ultrahigh Strain and Piezoelectric Behavior in Relaxor Based Ferroelectric Single Crystals. J Appl Phys 1997; 82[4]: 1804-11.

[5] Rödel J, Webber K G, Dittmer R, Jo W, Kimura M, Damjanovic D. Transferring lead-free piezoelectric ceramics into application. J Eur Ceram Soc 2015; 35: 1659-1681.

[6] Coondoo I, Panwar N, Kholkin A. Lead-free piezoelectrics: Current status and perspectives. J Adv Dielect 2013; 3: 1330002.

[7] Jaffe B, Cook W R, Jaffe H. Piezoelectric Ceramics 1971; Academic, New York.

[8] Budimir M, Damjanovic D, Setter N. Piezoelectric Anisotropy-Phase Transition Relations in Perovskite Single Crystals. J Appl Phys 2003; 94: 6753.

[9] Saito Y, Takao H, Tani T, Nonoyama T, Takatori K, Homma T,Nagaya T, Nakamura M. Lead-free piezoceramics. Nature 2004; 432: 84-7.

[10] Shrout T R, Zhang S J. Lead-free piezoelectric ceramics: Alternatives for PZT?. J Electroceram 2007; 19: 111-124.

[11] Damjanovic D. A morphotropic phase boundary system based on polarization rotation and polarization extension. Appl Phys Lett 2010; 97: 062906.

[12] Tani T, Kimura T. Reactive-Templated Grain Growth Processing for Lead Free Piezoelectric Ceramics. Adv Appl Ceram 2006; 105 [1]: 55-63.

[13] Wada S, Yako K, Kakemoto H, Tsurumi T, Kiguchi T. Enhanced Piezoelectric Properties of Barium Titanate Single Crystals with Different Engineered-Domain Sizes. J Appl Phys 2005; 98: 014109.

[14] Liu W, Ren X. Large Piezoelectric Effect in Pb-Free Ceramics. PRL 2009; 103: 257602.

[15] Keeble D S, Benabdallah F, Thomas P A, Maglione M, Kreisel J. Revised structural phase diagram of $\left(\mathrm{Ba}_{0.7} \mathrm{Ca}_{0.3} \mathrm{TiO}_{3}\right)-\left(\mathrm{BaZr}_{0.2} \mathrm{Ti}_{0.8} \mathrm{O}_{3}\right)$. Appl Phys Lett 2013; 102: 092903.

[16] Bao H, Zhou C, Xue D, Gao J, Ren X. A modified lead-free piezoelectric BZT-xBCT system with higher T. J Phys D: Appl Phys 2010; 43: 465401.

[17] Ye S K, Fuh J Y H, Lu L. Structure and electrical properties of $<001>$ textured $\left(\mathrm{Ba}_{0.85} \mathrm{Ca}_{0.15}\right)$ $\left(\mathrm{Ti}_{0.9} \mathrm{Zr}_{0.1}\right) \mathrm{O}_{3}$ lead-free piezoelectric ceramics. Appl Phys Lett 2012; 100: 252906.

[18] Deng X, Wang X, Wen H, Kang A, Gui Z, Li L. Phase Transitions in Nanocrystalline Barium Titanate Ceramics Prepared by Spark Plasma Sintering. J Am Ceram Soc 2006; 89[3]: 1059-1064.

[19] Deng X, Wang X, Wen H, Chen L, Chen L, Li L. Ferroelectric properties of nanocrystalline barium titanate ceramics. Appl Phys Lett 2006; 88: 252905.

[20] Hungria T, Alguero M, Hungria A B, Castro A. Dense, Fine-Grained $\mathrm{Ba}_{1-x} \mathrm{Sr}_{x} \mathrm{TiO}_{3}$ Ceramics Prepared by the Combination of Mechanosynthesized Nanopowders and Spark Plasma Sintering. Chem. Mater. 2005; 17: 6205-6212. 
[21] Li J-F, Wang K, Zhang B-P, Zhang L-M. Ferroelectric and Piezoelectric Properties of Fine-Grained $\mathrm{Na}_{0.5} \mathrm{~K}_{0.5} \mathrm{NbO}_{3}$ Lead-Free Piezoelectric Ceramics Prepared by Spark Plasma Sintering. J Am Ceram Soc 2006; 89[2]: 706-709.

[22] Shen Z-Y, Li J-F, Wang K, Xu S, Jiang W, Deng Q. Electrical and Mechanical Properties of FineGrained Li/Ta-Modified ( $\mathrm{Na}, \mathrm{K}) \mathrm{NbO}_{3}$-Based Piezoceramics Prepared by Spark Plasma Sintering. J Am Ceram Soc 2010; 93[5]: 1378-1383.

[23] Cernea M, Poli G, Aldica G V, Berbecaru C, Vasile B S, Galassi C. Preparation and properties of nanocrystalline BNT-BT $x$ piezoelectric ceramics by sol-gel and spark plasma sintering. Curr Appl Phys 2012; 12: 1100-1105.

[24] Deluca M, Vasilescu C A, lanculescu A C, Berger D C, Ciomaga C E, Curecheriu L P et al. Investigation of the composition-dependent properties of $\mathrm{BaTi}_{1-} \mathrm{Zr}_{\mathrm{x}} \mathrm{O}_{3}$ ceramics prepared by the modified Pechini method. J Eur Ceram Soc 2012; 32: 3551-3566

[25] Hao J, Bai W, Li W, Zhai J. Correlation Between the Microstructure and Electrical Properties in High-Performance $\left(\mathrm{Ba}_{0.85} \mathrm{Ca}_{0.15}\right)\left(\mathrm{Zr}_{0.1} \mathrm{Ti}_{0.9}\right) \mathrm{O}_{3}$ Lead-Free Piezoelectric Ceramics. J Am Ceram Soc 2012; 95[6]: 1998-2006.

[26] Tang X-G, Chan H L-W. Effect of grain size on the electrical properties of (Ba,Ca)(Zr,Ti) $\mathrm{O}_{3}$ relaxor ferroelectric ceramics. J Appl Phys 2005; 97: 034109.

[27] Tan Y, Zhang J, Wu Y, Wang C, Koval V, Shi B, Ye H, McKinnon R, Viola G, Yan H. Unfolding grain size effects in barium titanate ferroelectric ceramics. Scientif Reports 2015; 5:9953.

[28]IEEE Standard on Piezoelectricity,1987; ANSI/IEEE Std. 176.

[29] Zhang Y, Glaum J, Groh C, Ehmke M C, Blendell J E, Bowman K J, Hoffman M J. Correlation Between Piezoelectric Properties and Phase Coexistence in $(\mathrm{Ba}, \mathrm{Ca})(\mathrm{Ti}, \mathrm{Zr}) \mathrm{O}_{3}$ Ceramics. J Am Ceram Soc 2014; 97[9]: 2885-2891.

[30] Randall C A, Kim N, Kucera J-P, Cao W, Shrout T R. Intrinsic and Extrinsic Size Effects in FineGrained Morphotropic-Phase-Boundary Lead Zirconate Titanate Ceramics. J Am Ceram Soc 1998; 81[3]: 677-688.

[31] Stephens P W. Phenomenological model of anisotropic peak broadening in powder diffraction. J Appl Cryst 1999; 32: 281-289.

[32] Frey M H, Xu Z, Han P, Payne D A. The Role of Interfaces on an Apparent Grain Size Effect on the Dielectric Properties for Ferroelectric Barium Titanate Ceramics. Ferroelectrics 1998; 206-207: 33753. 
[33] Emelyanov A Y, Pertsev N A, Hoffman-Eifert S, BÖttger U, Waser R. Grain-Boundary Effect on the Curie-Weiss Law of Ferroelectric Ceramics and Polycrystalline Thin Films: Calculation by Method of Effective Medium. J Electroceram 2002; 9: 5-16.

[34] Wu Y J, Uekawa N, Kakegawa K, Sasaki Y. Compositional fluctuation and dielectric properties of $\mathrm{Pb}\left(\mathrm{Zr}_{0.3} \mathrm{Ti}_{0.7}\right) \mathrm{O}_{3}$ ceramics prepared by spark plasma sintering. Materials Letters 2002; 57: 771-775.

[35] Uchino K, Nomura S. Critical Exponents of the Dielectric Constants in Diffused Phase Transition Crystals. Ferroelectr Lett Sect 1982; 44: 55-61.

[36] Benabdallah F, Simon A, Khemakhem H, Elissalde C, Maglione M. Linking large piezoelectric coefficients to highly flexible polarization of lead free $\mathrm{BaTiO}_{3}-\mathrm{CaTiO}_{3}-\mathrm{BaZrO}_{3}$ ceramics. J Appl Phys 2011; 109: 124116.

[37] Zuo R, Granzow T, Lupascu D C, RÖdel J. PMN-PT Ceramics Prepared By Spark Plasma Sintering. J Am Ceram Soc 2007; 90[4]: 1101-1106.

[38] Wang R P, Xie R J, Sekiya T, Shimojo Y. Fabrication and characterization of potassium-sodium niobate piezoelectric ceramics by spark-plasma-sintering method. Mater Res Bull 2004; 39: 1709.

[39] Maiwa H. Structure and properties of $\mathrm{Ba}\left(\mathrm{Zr}_{0.2} \mathrm{Ti}_{0.8}\right) \mathrm{O}_{3}$ ceramics prepared by spark plasma sintering. J Mater Sci 2008; 43: 6385-6390.

[40] Orihara H, Hashimoto S, Ishibashi Y. A Theory of D-E Hysteresis Loop Based on the Avrami Model. J Phys Soc Jpn 1994; 63: 1031-5.

[41] Chandrasekaran A, Damjanovic D, Setter N, Marzari N. Defect ordering and defect-domain-wall interactions in PbTiO3: A first-principles study. Phys Rev B 2013; 88: 214116.

[42] Rabe K M, Ahn C H, Triscone J- M. Physics of ferroelectrics a modern perspective 2007; SpringerVerlag Berlin Heidelberg.

[43] Sherrit S, Wiederick H D, Mukherjee B K, Sayer M. An accurate equivalent circuit for the unloaded piezoelectric vibrator in the thickness mode. J Phys D: Appl Phys 1997; 30: 2354-2363.

[44] Martin G. Determination of equivalent circuit constants of piezoelectric resonators of moderately low $Q$ by absolute-admittance measurements. J Acoust Soc Am 1954; 26: 413-20.

[45] Ikeda T. Fundamentals of Piezoelectricity 1990; Oxford:Oxford University Press, p 140.

[46] Terunuma K, Nishigaki S. A method for measuring the equivalent circuit elements for a piezoresonator. Jpn J Appl Phys 1983; 22: suppl 22-2 143-5.

[47] Xue D, Zhou Y,Bao H, Zhou C, Gao J, Ren X. Elastic, piezoelectric, and dielectric properties of $\mathrm{Ba}\left(\mathrm{Zr}_{0.2} \mathrm{Ti}_{0.8}\right) \mathrm{O}_{3}-50\left(\mathrm{Ba}_{0.7} \mathrm{Ca}_{0.3}\right) \mathrm{TiO}_{3} \mathrm{~Pb}$-free ceramic at the morphotropic phase boundary. J Appl Phys 2011; 109: 054110.

[48] Praveen J P, Karthik T, James A R, Chandrakala E, Asthana S, Das D. Effect of poling process on piezoelectric properties of sol-gel derived BZT-BCT ceramics. J Eur Ceram Soc 2015; 35: 1785-1798. 
[49] Gao J, Xue D, Wang Y, Wang D, Zhang L et al. Microstructure basis for strong piezoelectricity in $\mathrm{Pb}$-free $\mathrm{Ba}\left(\mathrm{Zr}_{0.2} \mathrm{Ti}_{0.8}\right) \mathrm{O}_{3}-\left(\mathrm{Ba}_{0.7} \mathrm{Ca}_{0.3}\right) \mathrm{TiO}_{3}$ ceramics. Appl Phys Lett 2011; 99: 092901.

[50] Curecheriu L, Balmus S-B, Buscaglia M T, Buscaglia V, lanculescu A, Mitoseriu L. Grain SizeDependent Properties of Dense Nanocrystalline Barium Titanate Ceramics. J Am Ceram Soc 2012; 95[12]: 3912-3921. 

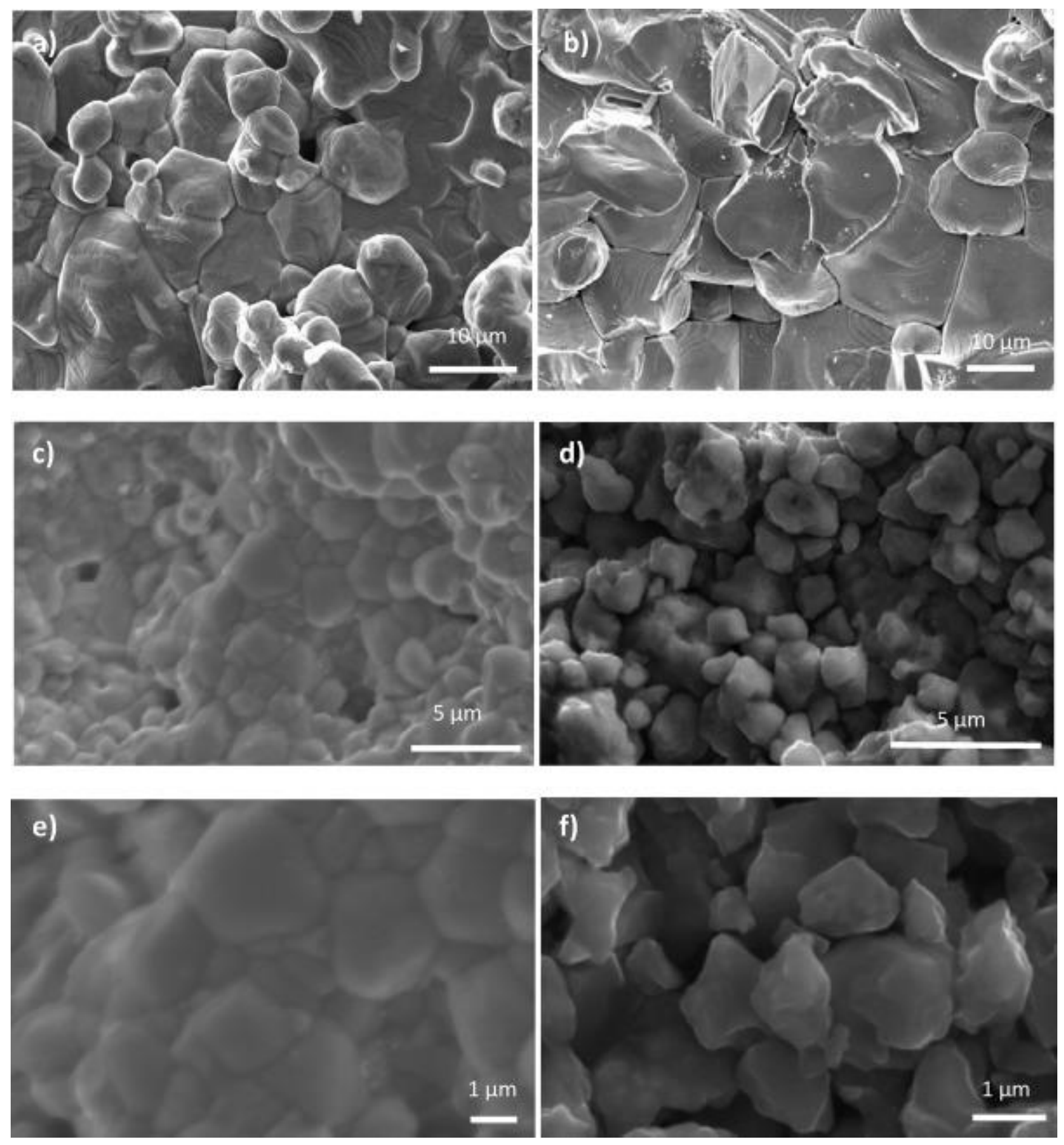

Fig. 1. Scanning electron microscope (SEM) micrographs of fracture surfaces: (a) BCTZ32 $(x=0.32)$ and (b) BCTZ50 ( $x=0.5$ ) ceramics sintered at $1450{ }^{\circ} \mathrm{C} / 4 \mathrm{~h} / \mathrm{O}_{2}$; (c, e) BCTZ32 (d, f) BCTZ50 sintered at $1300{ }^{\circ} \mathrm{C} / 90 \mathrm{MPa} / 10$ min using spark plasma sintering (after thermal and chemical etching respectively). 

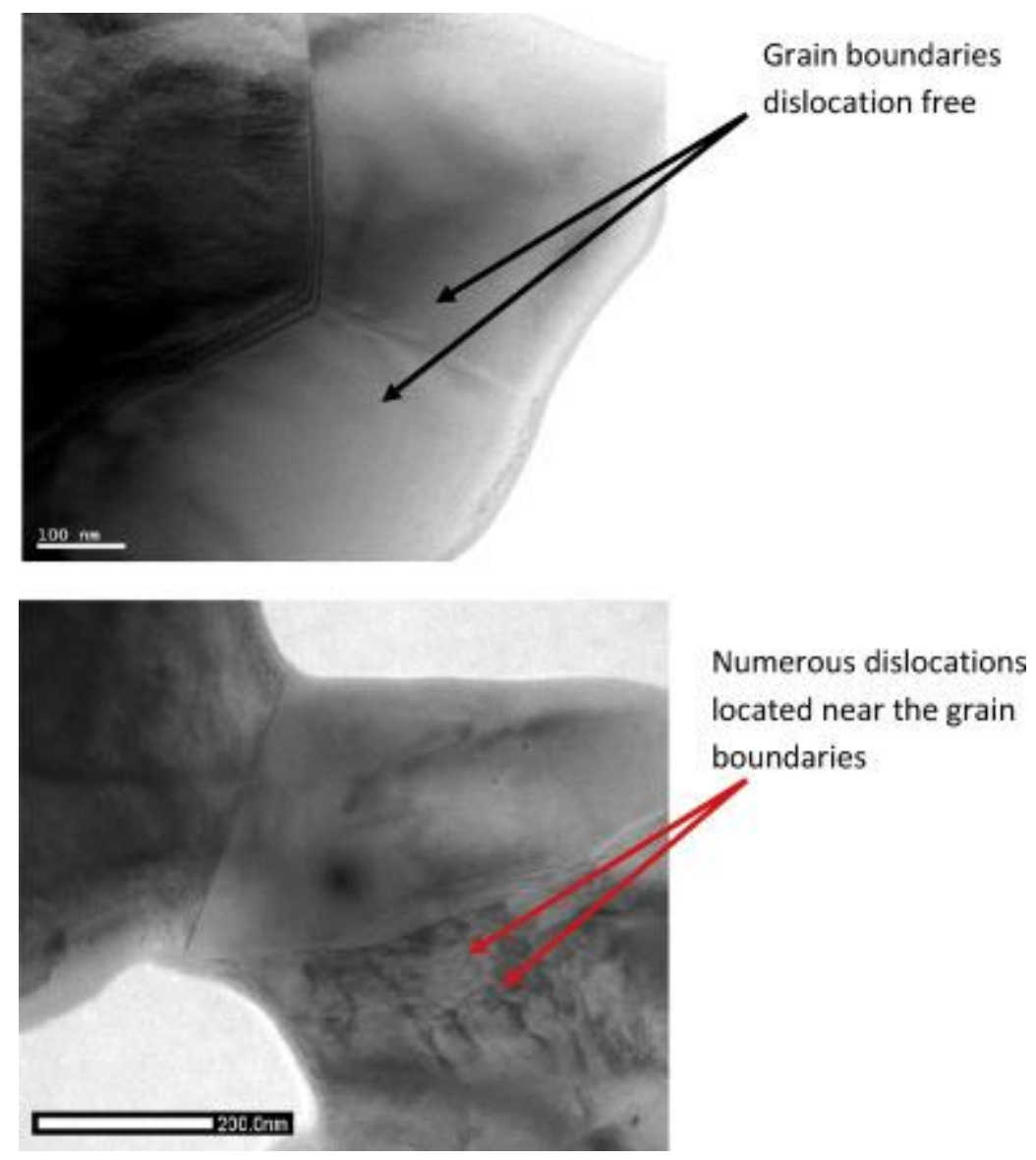

Fig. 2. Transmission electron microscopy (TEM) images of SPS processed BCTZ ceramic $(x=0.32$, $\left.1300^{\circ} \mathrm{C} / 90 \mathrm{MPa} / 10 \mathrm{~min}\right)$ : the images correspond to the surfaces of thin plates explored perpendicular to the SPS pressing direction. The presence of numerous dislocations within some individual grains is noticed close to grain boundaries. This finding proves that the use of selected parameters during the SPS process can provoke both nonrecoverable and irreversible plastic deformations on grain surfaces. 

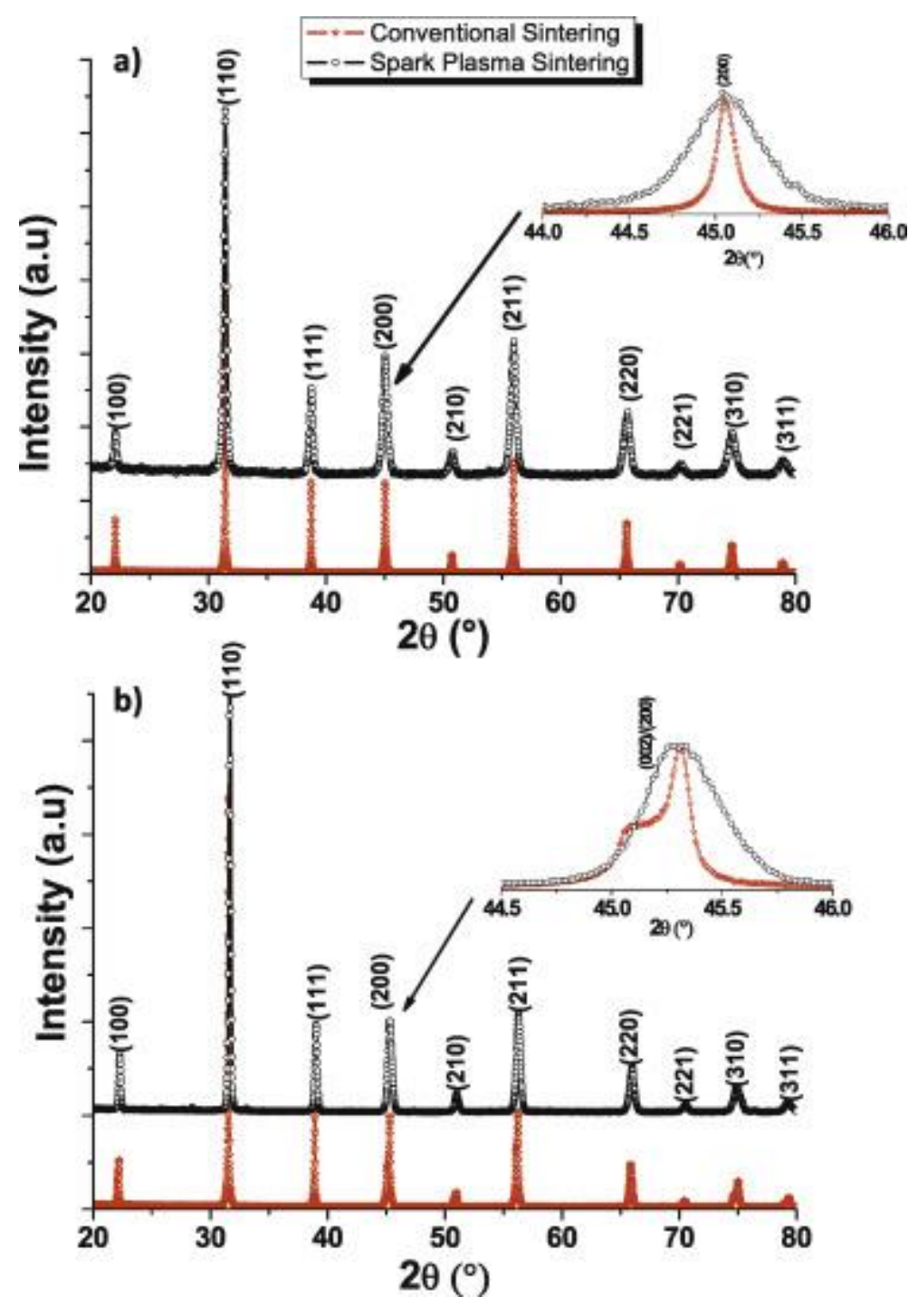

Fig. 3. Standard X-ray diffraction patterns of $B C T Z$ ceramics prepared under different sintering conditions: (a) BCTZ32, (b) BCTZ50. Inset: zoom on the diffraction profiles around $2 \theta \approx 45^{\circ}$. 


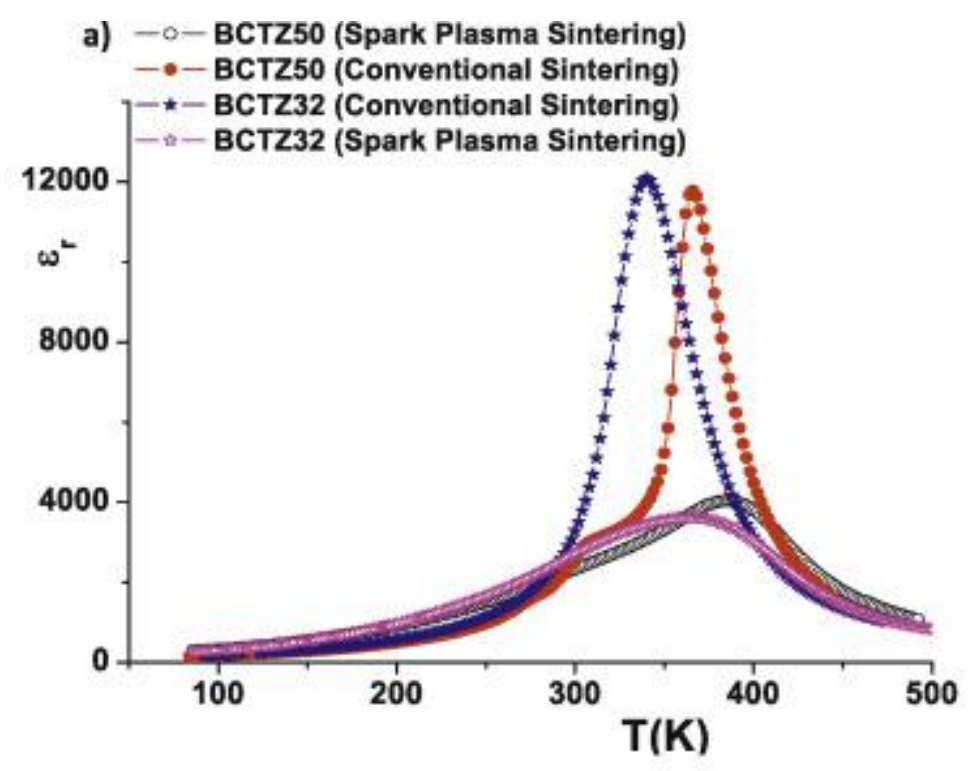

b)

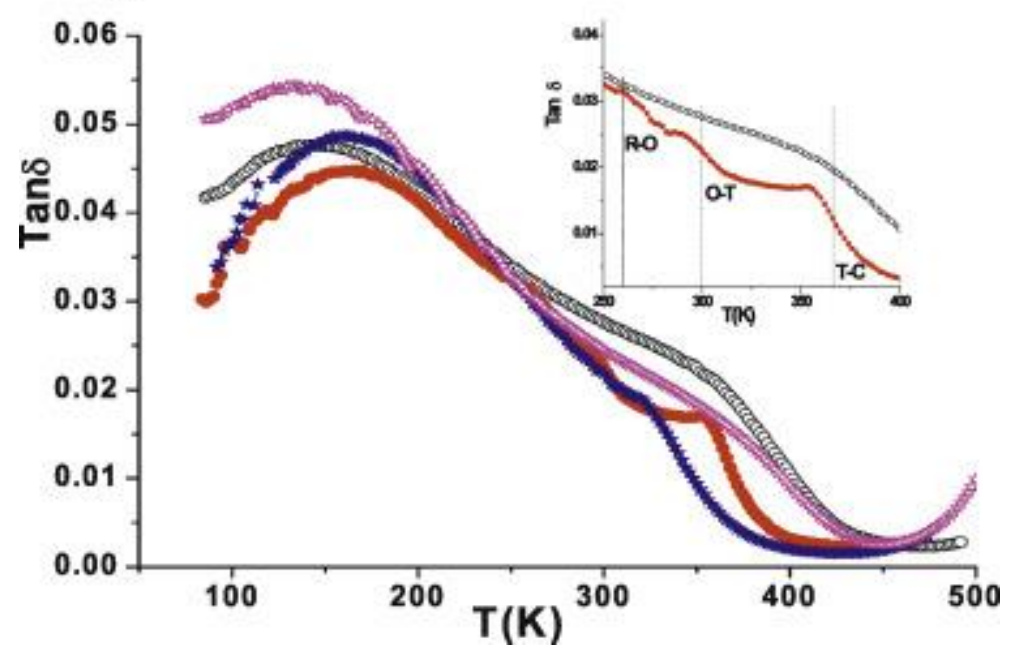

Fig. 4. Temperature dependence of the dielectric properties of BCTZ ceramics prepared under different sintering conditions: the conventional sintering was done at $1450{ }^{\circ} \mathrm{C} / 4 \mathrm{~h}$ in oxygen atmosphere and the spark plasma sintering performed at $1300{ }^{\circ} \mathrm{C} / 90 \mathrm{MPa} / 10 \mathrm{~min}$. (a) The relative dielectric permittivity and (b) loss tangent $(\tan \delta)$ recorded at $10 \mathrm{kHz}$. Inset: focus on the well defined $\mathrm{R}-\mathrm{O}$ and $\mathrm{O}-\mathrm{T}$ phase transitions in conventionally sintered BCTZ50 which disappear in the SPS processed ceramic. 

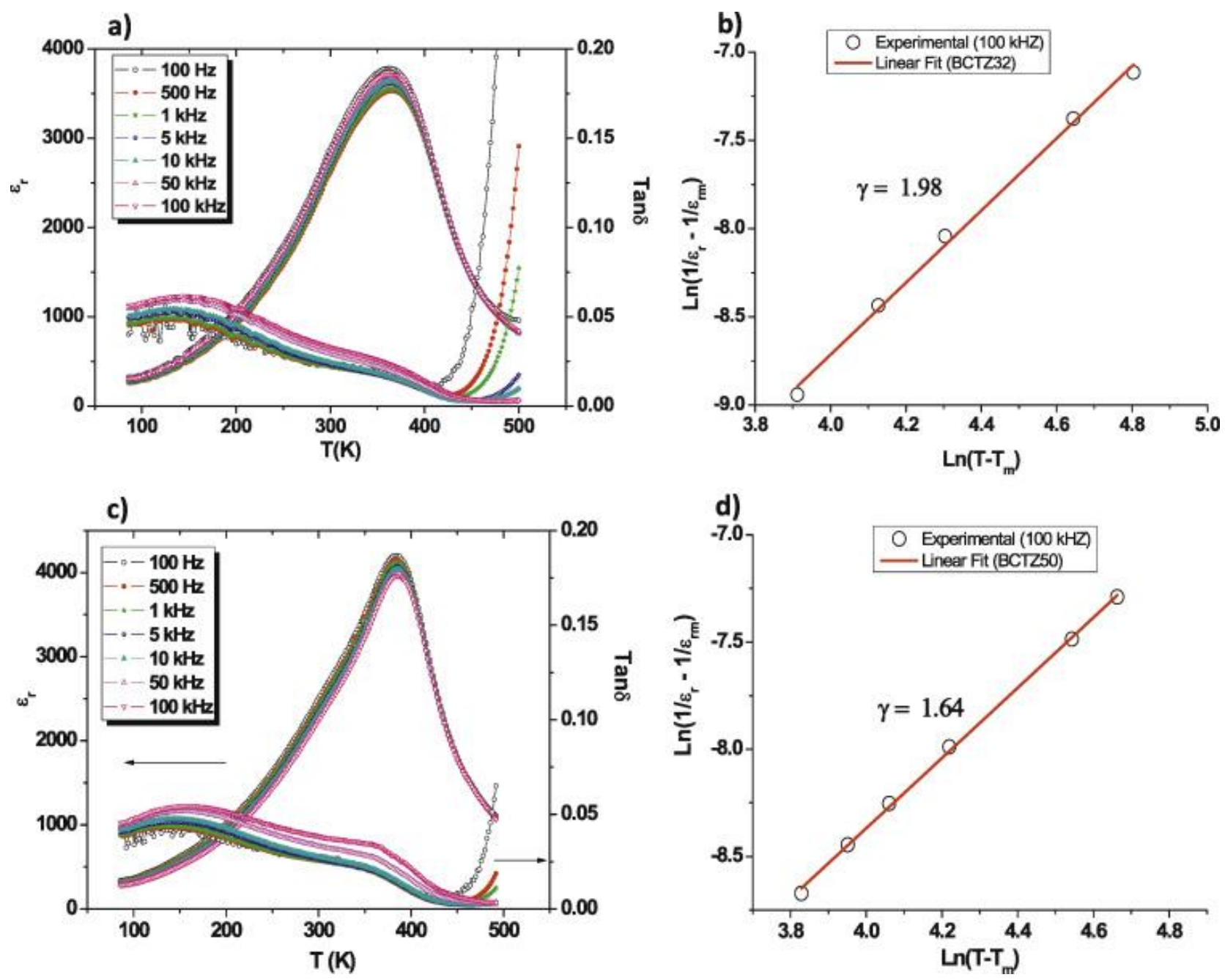

Fig. 5. (a, c) Temperature dependence of the dielectric constant $\epsilon_{r}$ and loss tangent ( $\tan \delta$ ) for SPS processed BCTZ32 and BCTZ50, respectively. The measurements were recorded at different frequencies ranging between $10^{2}$ and $10^{5} \mathrm{~Hz}$. $(\mathrm{b}, \mathrm{d})$ : $\ln \left(1 / \epsilon_{\mathrm{r}}-1 / \epsilon_{\mathrm{rm}}\right)$ as a function of $\ln \left(T-T_{\mathrm{m}}\right)$ for the BCTZ ceramics prepared using SPS at $100 \mathrm{kHz}\left(T>T_{\mathrm{m}}\right)$. The values of $\gamma$ calculated from the slope of the graphs reflect the increase of the relaxor features from BCTZ50 $(y=1.64)$ to BCTZ32 $(x=0.32)$

$$
(\gamma=1.98) \text {. }
$$




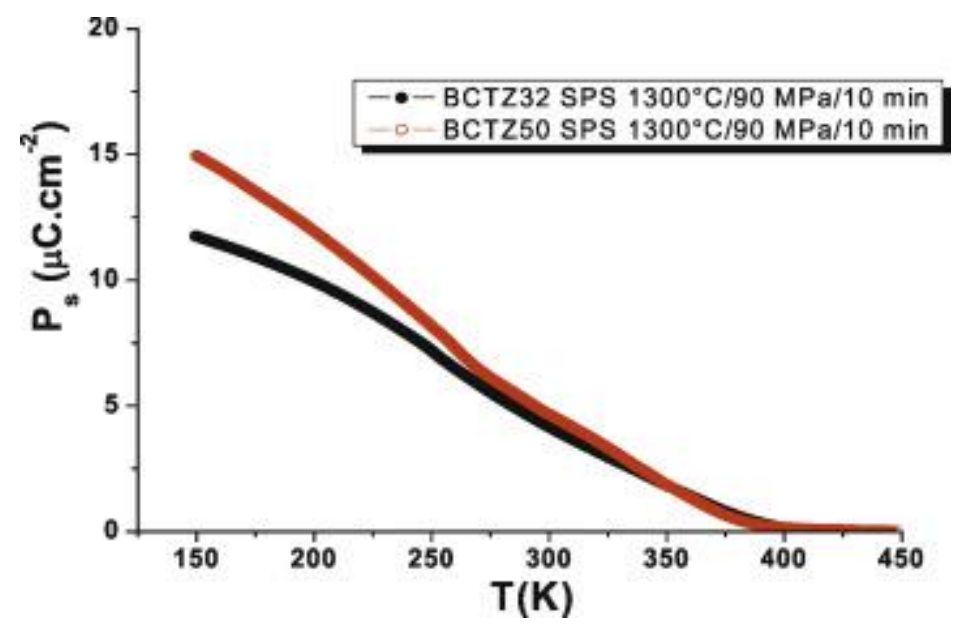

Fig. 6. Temperature dependence of the spontaneous polarization Ps in BCTZ ceramics $(x=0.32$ and $0.5)$ processed by SPS.

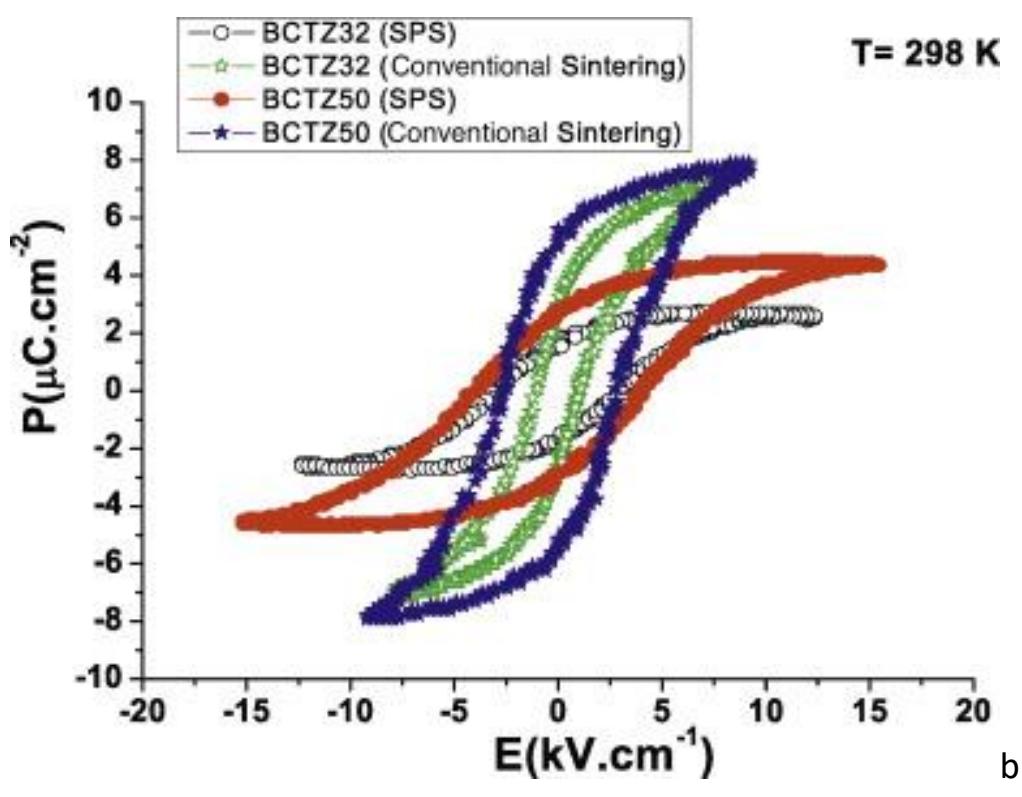

Fig. 7. Polarization versus electric field $P(E)$ hysteresis loops of $B C T Z$ ceramics (BCTZ32 $(x=0.32)$ and $\mathrm{BCTZ50}(x=0.5))$ prepared using conventional and spark plasma sintering methods. The measurements were performed in dynamic regime at $50 \mathrm{~Hz}$ and $T=298 \mathrm{~K}$. 

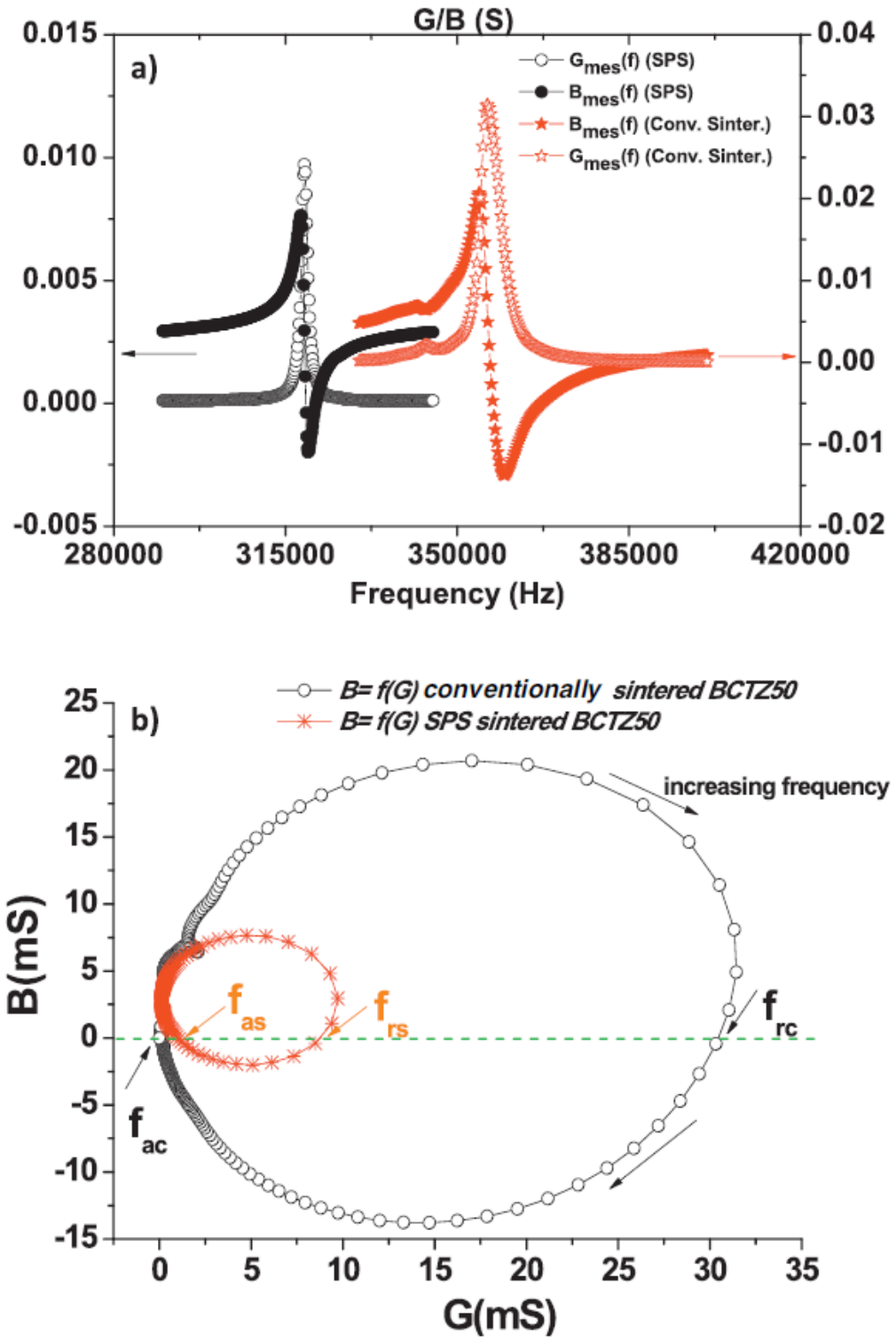

Fig. 8. (a) Plots of the conductance $G$ and the susceptance B measured for conventionally and SPS sintered BCTZ50 ceramic discs for the radial vibration mode at room temperature. (b) The admittance locus obtained for BCTZ50 ceramic discs prepared using conventional and spark plasma sintering methods: the experimental data points shown as open circles are generated by the normal sintered sample while those plotted as red stars correspond to the SPS sintered one. $\left(f_{\mathrm{rc}}, f_{\mathrm{rs}}\right),\left(f_{\mathrm{ac}}\right.$, $f_{\text {as }}$ ) refer to the frequencies of resonance and the frequencies of antiresonance of the studied samples respectively $\left(f_{\mathrm{r}} \approx f_{\mathrm{s}}\right.$ the series resonance frequency, $f_{\mathrm{a}} \approx f_{\mathrm{p}}$ the parallel resonance frequency). The ratio $\frac{f_{\mathrm{p}}-f_{\mathrm{s}}}{f_{\mathrm{S}}} \approx \frac{f_{\mathrm{a}}-f_{\mathrm{r}}}{f_{\mathrm{r}}}$ determines the strength of the electro-mechanical coupling. 